\title{
AIDER UN PARENT ÂGÉ SE FAIT-IL AU DÉTRIMENT DE L'EMPLOI ? Roméo FONTAINE
}

\author{
La Documentation française | « Retraite et société »
}

2009/2 n 58 | pages 31 à 61

ISSN 1167-4687

Article disponible en ligne à l'adresse :

http://www.cairn.info/revue-retraite-et-societe-2009-2-page-31.htm

\section{Pour citer cet article :}

Roméo FONTAINE, «Aider un parent âgé se fait-il au détriment de l'emploi ? 》, Retraite et société 2009/2 (n58), p. 31-61.

Distribution électronique Cairn.info pour La Documentation française.

(c) La Documentation française. Tous droits réservés pour tous pays.

La reproduction ou représentation de cet article, notamment par photocopie, n'est autorisée que dans les limites des conditions générales d'utilisation du site ou, le cas échéant, des conditions générales de la licence souscrite par votre établissement. Toute autre reproduction ou représentation, en tout ou partie, sous quelque forme et de quelque manière que ce soit, est interdite sauf accord préalable et écrit de l'éditeur, en dehors des cas prévus par la législation en vigueur en France. Il est précisé que son stockage dans une base de données est également interdit. 
Aider un parent

âgé se fait-il au

détriment de

l'emploi? 
Le vieillissement de la population apparaît en Europe comme un des principaux enjeux des prochaines décennies. Il fait craindre dans la majorité des pays une pression difficilement soutenable sur les systèmes publics de protection sociale. Afin de relever ce défi démographique, le Conseil européen de Stockholm (2001) a donné pour objectif aux États membres de I'Union européenne (UE) de porter le taux d'emploi à une moyenne européenne de $67 \%$, en fixant des objectifs particuliers pour la population dite des seniors. Il a en effet été « convenu de se fixer pour I'horizon 2010 l'objectif de porter à 50 \% le taux d'emploi moyen dans I'UE pour la catégorie d'âge, hommes et femmes confondus, de 55 à 64 ans $^{1}$ ». Cet objectif des $50 \%$ fut par la suite renouvelé par le programme communautaire de Lisbonne (2005). Le graphique 1 présente la situation en 2008 des différents pays présents dans l'enquête Share. En France, le taux d'emploi des seniors, hommes et femmes confondus, apparaît en particulier comme l'un des plus faibles d'Europe.

Incitée à travailler, il semble que la population des seniors soit aussi incitée à soutenir les nombreuses personnes, en particulier leurs parents, nécessitant une prise en charge dans la réalisation des activités de la vie quotidienne. La thématique de I' " aide aux aidants », qui vise à " trouver plusieurs voies pour accompagner et soutenir cet engagement solidaire au sein des familles " (Conférence de la Famille, 2006), semble illustrer dans le cas français cette volonté politique.

La question est alors de savoir si une politique visant à prolonger l'activité des seniors est compatible avec une politique visant à entretenir l'aide informelle dont peuvent bénéficier les personnes âgées nécessitant une prise en charge. Ne peut-on pas craindre une diminution de l'aide informelle en cas d'augmentation du taux d'emploi des seniors? Ou au contraire, faire essentiellement reposer la prise en charge des personnes âgées sur les familles n'est-il pas un frein à l'augmentation du taux d'emploi des seniors?

L'enquête Share apporte des outils précieux pour fournir des éléments de réponses à ces questions car elle permet une comparaison entre des pays dont le contexte institutionnel et culturel varie de manière assez 
importante ${ }^{2}$. Dans certains pays, en particulier dans les pays d'Europe du Nord, la prise en charge des personnes âgées dépendantes repose en grande partie sur des mécanismes de solidarités publiques, ce qui limite a priori les effets négatifs de l'aide informelle sur le taux d'emploi. C'est par exemple le cas de la Suède qui consacrait en 2004 près de 2,6 \% de son PIB aux dépenses de soins de long terme et où près de $7 \%$ des plus de 65 ans vivaient en institution au début des années 1990 (Bolin et al., 2008). Dans d'autres pays, en particulier dans les pays d'Europe du Sud, les dispositifs de prise en charge publique sont moins importants. L'Espagne consacrait par exemple en 2004 près de $0,3 \%$ de son PIB aux soins de longue durée alors que moins de $3 \%$ de la population des plus de 65 ans vivaient en institution (Bolin et al., 2008). Les pays d'Europe du Sud sont dès lors généralement décrits comme des pays où l'aide aux personnes âgées dépendantes repose essentiellement sur les familles. Les enfants, principaux aidants informels lorsque la personne âgée ne peut plus compter sur le soutien d'un conjoint, pourraient ainsi être contraints plus qu'ailleurs d'arbitrer entre offre d'aide et offre de travail.

\section{Graphique 1. Taux d'emploi en 2008 des seniors (55-64 ans) des pays représentés dans l'enquête Share}

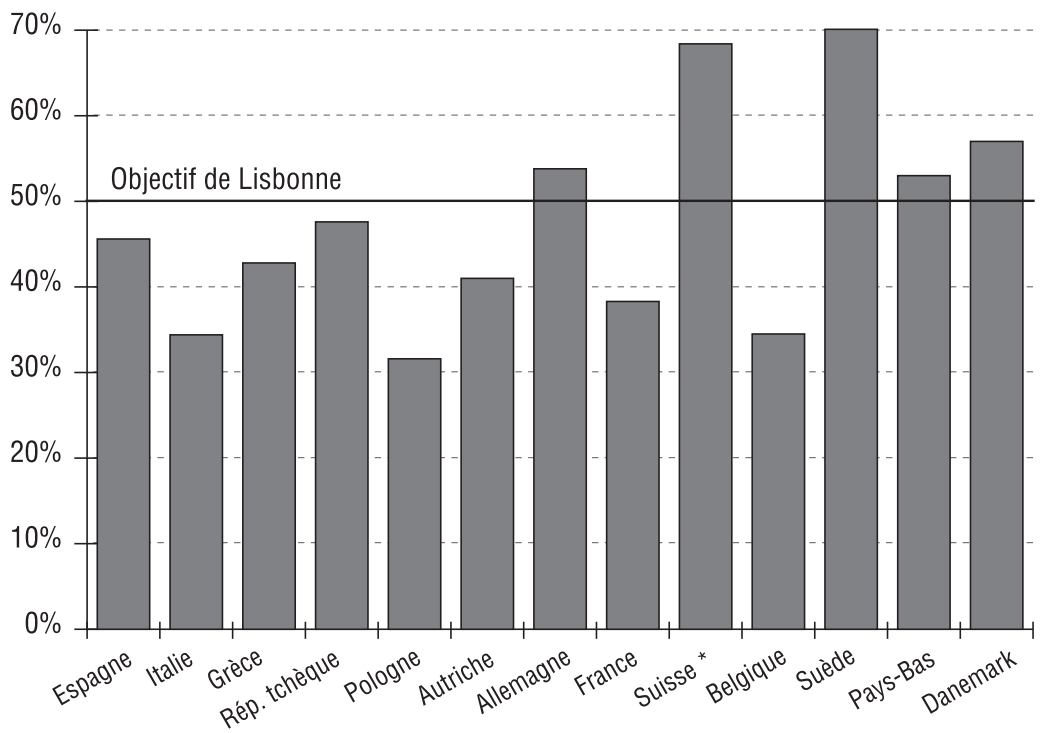

Source : Eurostat.

* N'étant pas membre de l'UE, la Suisse n'est pas concernée par l'objectif de Lisbonne.

Le graphique 2 (page 34) illustre à I'aide des données de l'enquête Share I'hétérogénéité des pays européens. Ce graphique rapproche le taux d'emploi des femmes âgées de 50 à 65 ans de la proportion d'entre elles

2 Se reporter au rapport de l'OCDE (2005) pour une comparaison des systèmes publics de prise en charge de la dépendance. 
qui apportent plus d'une heure d'aide par jour à leur parent âgé ou qui cohabitent avec ce dernier ${ }^{3}$. Une relation décroissante apparaît entre emploi et aide. À l'une des extrémités se situent les pays d'Europe du Nord et la Suisse, caractérisés par un taux d'emploi des femmes élevé et une faible proportion de femmes aidant plus d'une heure par jour ou cohabitant avec un parent âgé. À l'autre extrémité se trouvent les pays d'Europe du Sud et d'Europe de l'Est, caractérisés par un faible taux d'emploi et une forte proportion de femmes aidant plus d'une heure par jour ou cohabitant avec un parent âgé. Les autres pays se situent quelque part entre les deux. À noter que la France a un profil nettement plus proche de celui des pays nordiques que des pays d'Europe du Sud, ce qui constitue un résultat assez fréquent lorsque l'on compare en Europe l'aide des enfants à leurs parents âgés.

Avant d'aller plus loin dans l'analyse des effets de l'aide informelle sur l'exercice d'une activité professionnelle, nous présenterons rapidement la population que nous proposons d'étudier ici et mènerons une rapide comparaison à travers l'Europe de l'aide apportée par les seniors à leur parent âgé.

\section{Graphique 2. Emploi et aide au sein de la population des femmes âgées de 50 à 65 ans n'ayant plus qu'un parent en vie}

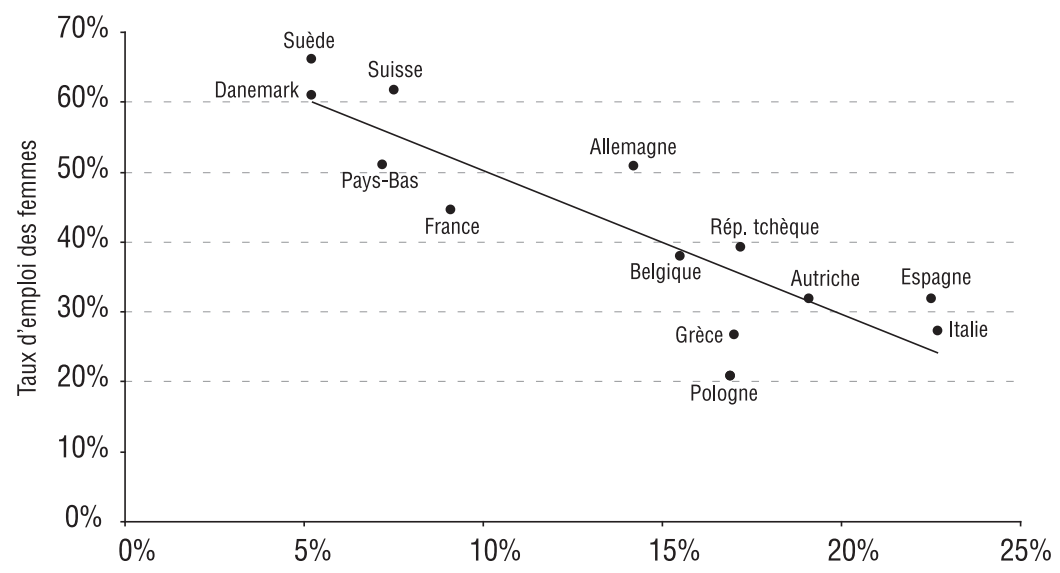

Proportion de femmes aidant plus d'une heure par jour ou cohabitant avec un parent âgé

Lecture : en Suède, le taux d'emploi des femmes âgées de 50 à 65 ans est de 66,13\%. Elles sont, par ailleurs, parmi celles n'ayant plus qu'un parent en vie, $5,20 \%$ à aider plus d'une heure par jour leur parent ou à cohabiter avec ce dernier.

Champ : femmes âgées de 50 à 65 ans.

Source : Eurostat et enquête Share, vague 2 (2006-2007).

3 Nous nous concentrons ici, comme dans la suite de l'analyse, sur l'aide que les seniors apportent à un parent seul, c'est-à-dire ne pouvant plus compter sur l'aide d'un conjoint. 


\section{Population étudiée}

Afin d'étudier de quelle manière les seniors articulent aide informelle et emploi, nous avons restreint l'échantillon Share aux individus âgés de 50 à 65 ans, les individus au-delà de cet âge n'étant que trop rarement actifs. L'analyse est ici centrée sur l'aide informelle qu'apportent les seniors à leurs parents. Une autre possibilité aurait été d'étudier l'aide apportée par les conjoints des personnes âgées, mais les conséquences de cette aide sur le marché de l'emploi sont moins attendues étant donné qu'elle concerne généralement des individus plus âgés, et donc en grande majorité déjà sortis du marché du travail. L'échantillon est par ailleurs restreint aux individus n'ayant au moment de l'enquête plus qu'un parent en vie. L'aide apportée par les enfants varie en effet considérablement suivant que le parent âgé ayant besoin d'une aide peut ou non compter sur la présence d'un conjoint. Quand un conjoint est présent, la mobilisation des enfants est semble-t-il moins importante, si bien que la question de l'articulation entre emploi et aide apparaît dans ce cas moins pertinente 4 . Le graphique 3 (page 36) présente par âge la proportion d'individus ayant au moins un parent en vie au moment de l'enquête. À 50 ans, les Européens sont près de $60 \%$ à avoir au moins un parent en vie, la majorité d'entre eux n'ayant cependant plus qu'un parent en vie. Ils ne sont plus que $19 \%$ à avoir au moins un parent en vie à 60 ans et $2 \%$ à 70 ans. Les individus ayant plus de 65 ans ne sont donc que dans une relativement faible proportion concernés par l'aide à un parent, ce qui apporte une autre justification au fait d'exclure de l'échantillon étudié les individus au-delà de cet âge. Au final, en ne conservant que les individus de 50 à 65 ans et ayant un seul parent en vie au moment de I'enquête, l'échantillon utilisé ici comprend 4735 observations.

Les données utilisées sont celles de la deuxième vague de l'enquête Share. Treize pays y sont représentés. Nous distinguerons dans la suite de I'analyse autant que possible ces treize pays. Cependant, la taille des échantillons nationaux, en particulier lorsque nous identifierons les individus apportant une aide " intensive » à leur parent âgé, se révélera parfois trop réduite pour faire des comparaisons statistiques. Nous serons alors contraints de comparer des groupes de pays. Cette pratique est assez courante.

À I'instar de Bonsang (2007), Bolin et al. (2008), Casado-Marín et al. (2008) ou Crespo (2007), nous distinguerons (i) les pays d' "Europe continentale » rassemblant la France, I'Allemagne, I'Autriche, la Belgique et la Suisse, (ii)

4 Se reporter à Fontaine et al. (2007) pour une comparaison de l'aide apportée par les enfants à leurs parents âgés dépendants suivant la présence ou non d'un conjoint. 
les pays d' "Europe du Nord » regroupant le Danemark, les Pays-Bas et la Suède, (iii) les pays d' «Europe du Sud » avec l'Espagne, la Grèce et l'Italie et enfin (iv) les pays d' «Europe de l'Est», correspondant à la Pologne et à la République tchèque, nouveaux entrants dans I'enquête Share.

\section{Graphique 3. Proportion d'individus ayant un ou deux parents en vie au moment de l'enquête}

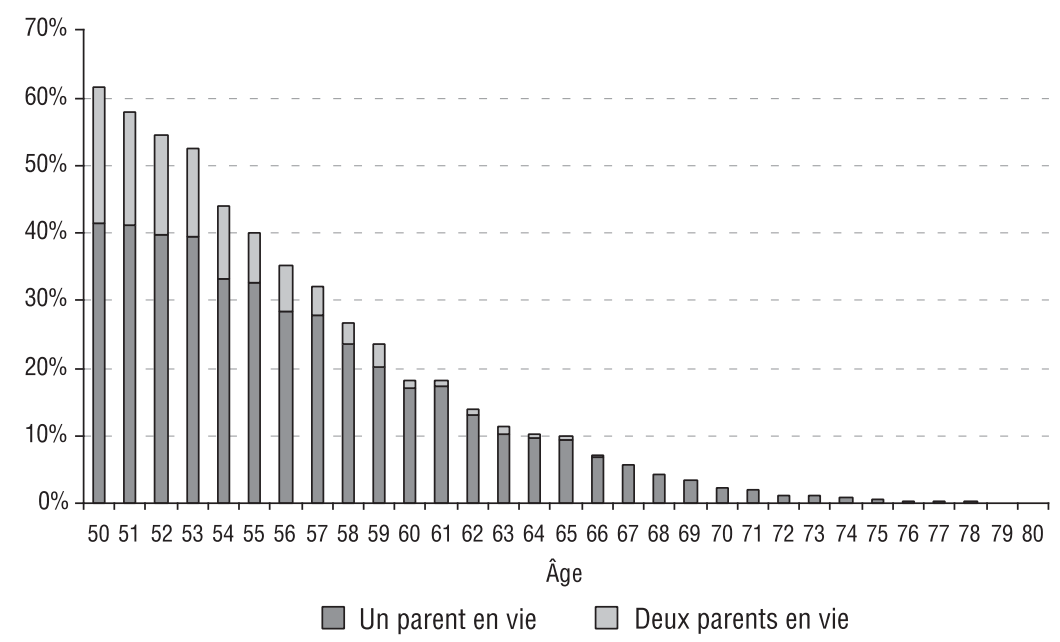

Lecture : parmi les Européens de 50 ans, $41 \%$ ont un parent en vie et $20 \%$ ont leurs deux parents en vie.

\section{Regard sur l'aide fournie par les seniors à leur parent âgé}

Avant d'étudier comment les seniors articulent aide et emploi, nous présentons ici une rapide comparaison à travers l'Europe de l'aide qu'apportent les individus de 50 à 65 ans à leur parent.

Le questionnaire de I'enquête Share comporte un certain nombre de questions permettant d'étudier l'aide que les seniors européens apportent à l'entourage ou en recoivent sous forme de service ${ }^{5}$. Les individus enquêtés sont interrogés sur trois types d'aide: l'aide dans les soins

5 Un autre volet de l'enquête, non utilisé ici, permet d'étudier les aides sous forme financière. La grande majorité des transferts intrafamiliaux ascendants se fait cependant sous forme de services. Les transferts financiers sont généralement des transferts descendants (Wolff \& Attias-Donfut, 2007). 
personnels (s'habiller, se laver, manger, se mettre au lit ou se lever, aller aux toilettes...), I'aide domestique (tâches ménagères, courses, déplacements, bricolage, jardinage...) et l'aide administrative (remplir des formulaires, régler des questions financières ou juridiques... $)^{6}$.

Le questionnaire n'aborde pas de la même manière l'aide informelle suivant qu'elle est fournie à des individus cohabitants ou à des individus " hors ménage ». Le questionnaire aborde l'aide interménage, c'est-à-dire impliquant deux individus appartenant à deux ménages distincts, à travers les trois types d'aide. En revanche, le questionnaire aborde I'aide intraménage, c'est-à-dire impliquant deux individus appartenant à un même ménage, uniquement à travers les soins personnels, et ne tient donc pas compte de l'aide domestique et de l'aide administrative. La question de I'intensité de l'aide (fréquence de l'aide et nombre d'heures d'aide apportée) n'est de plus abordée que pour l'aide interménage. Ces limitations concernant l'aide intraménage s'expliquent en grande partie par la difficulté à distinguer pour les individus enquêtés cohabitant avec un parent âgé ce qui relève d'une véritable aide de ce qui relève d'une organisation domestique indépendante des difficultés physiques ou cognitives des uns et des autres.

À l'instar de Fontaine et al. (2007), Ettner (1995, 1996) ou Heitmueller (2007), nous considérerons par la suite comme aidants, (i) I'ensemble des enfants ne cohabitant pas avec leur parent âgé mais déclarant lui apporter une aide dans les soins personnels, dans les activités domestiques ou administratives et (ii) I'ensemble des enfants cohabitants. Bien que nous n'ayons pas d'informations précises à leur sujet, nous considérons comme aidants l'ensemble des cohabitants étant donné qu'il est fort probable qu'ils apportent d'une manière ou d'une autre, au moins occasionnellement, une aide à leur parent âgé. La cohabitation semble par ailleurs une réponse à la dépendance d'un parent âgé sans conjoint, en particulier dans les pays du sud de l'Europe (Fontaine et al., 2007). Lorsque nous aborderons la question de l'intensité de l'aide, nous serons cependant contraints d'exclure de l'analyse les enfants cohabitants puisque nous n'avons aucune information sur l'intensité de leur aide. Enfin, parmi les enfants apportant une aide hors ménage, nous qualifierons par la suite d'aidants « intensifs » ceux apportant plus d'une heure d'aide par jour à leur parent âgé.

6 II convient de noter que nous nous concentrons ici uniquement sur les «aides productives ", c'est-à-dire les aides s'inscrivant dans une production effective de prise en charge. Nous excluons donc de l'analyse deux dimensions de l'aide qui, bien que difficilement mesurables, semblent des éléments essentiels de la prise en charge. La première est celle correspondant au soutien psychologique que les enfants peuvent apporter par exemple grâce à leurs visites ou leurs coups de téléphone. La deuxième est celle traduisant la «charge mentale» qui pèse sur les aidants. La prise en charge d'un parent âgé mobilise en effet, en plus des ressources physiques et parfois financières, d'importantes ressources morales, surtout lorsque le parent présente des incapacités sévères. 


\section{Des seniors plus fréquemment impliqués dans l'aide à leur parent dans le nord de l'Europe}

Au sein de l'échantillon, près d'un tiers des enfants apportent une aide à leur parent âgé. La proportion d'enfants aidants n'est cependant pas identique dans tous les pays d'Europe. C'est dans les pays d'Europe du Nord que les enfants sont le plus fréquemment aidants. Ils seraient en effet près de $40 \%$ à déclarer aider leur parent âgé alors qu'ils ne seraient que $24 \%$ dans les pays d'Europe du Sud et d'Europe de l'Est, les pays d'Europe continentale étant dans une situation intermédiaire. Nous verrons par la suite que le gradient Nord-Sud s'inverse lorsque I'on tient compte de I'intensité de l'aide.

Dans plus de 9 cas sur 10, I'aide est une aide « hors ménage » (tableau 1), c'est-à-dire apportée par un enfant non-cohabitant. La cohabitation intergénérationnelle entre les seniors et leur parent est quasi inexistante dans les pays d'Europe du Nord. Elle est en revanche assez fréquente en Europe du Sud, où l'aide ascendante passe dans plus d' 1 cas sur 10 par la cohabitation. La situation de l'Espagne est de ce point de vue tout à fait particulière en Europe, car l'aide des seniors à leur parent passe dans près d'un cas sur deux par la formation d'un ménage intergénérationnel.

\section{Tableau 1. Proportion d'enfants aidants par pays}

\begin{tabular}{l|c|c|c} 
& \multicolumn{3}{|c}{ Proportion d'aidants } \\
& Proportion totale & Aidants hors ménage & Cohabitants \\
\hline Europe du Sud & $24 \%$ & $80 \%$ & $20 \%$ \\
\hline Espagne & $25 \%$ & $54 \%$ & $46 \%$ \\
\hline Italie & $30 \%$ & $88 \%$ & $12 \%$ \\
\hline Grèce & $19 \%$ & $86 \%$ & $14 \%$ \\
\hline Europe de l'Est & $24 \%$ & $92 \%$ & $8 \%$ \\
\hline République tchèque & $29 \%$ & $94 \%$ & $6 \%$ \\
\hline Pologne & $18 \%$ & $86 \%$ & $14 \%$ \\
\hline Europe continentale & $33 \%$ & $95 \%$ & $5 \%$ \\
\hline Autriche & $25 \%$ & $92 \%$ & $8 \%$ \\
\hline Allemagne & $35 \%$ & $93 \%$ & $7 \%$ \\
\hline France & $29 \%$ & $97 \%$ & $3 \%$ \\
\hline Suisse & $30 \%$ & $97 \%$ & $3 \%$ \\
\hline Belgique & $39 \%$ & $94 \%$ & $6 \%$ \\
\hline Europe du Nord & $41 \%$ & $99 \%$ & $1 \%$ \\
\hline Suède & $42 \%$ & $99 \%$ & $1 \%$ \\
\hline Pays-Bas & $38 \%$ & $100 \%$ & $0 \%$ \\
\hline Danemark & $42 \%$ & $99 \%$ & $1 \%$ \\
\hline Ensemble & $32 \%$ & $93 \%$ & $7 \%$ \\
\hline
\end{tabular}

Lecture : en Espagne, 25\% des enfants déclarent aider leur parent âgé. Dans 54\% des cas, l'aide correspond à une aide " hors ménage » et dans 46\% à une aide à l'intérieur du ménage (cohabitation). Champ : individus de 50 à 65 ans ayant un seul parent en vie.

Source : enquête Share, vague 2 (2006-2007). 


\section{Une intensité de l'aide plus importante dans le sud}

Les chiffres précédents présentent une situation prenant à contre-pied le traditionnel gradient Nord-Sud: comparativement à la France, I'aide des seniors à leur parent âgé serait plus fréquente dans le nord de l'Europe et moins fréquente dans le sud. Ce paradoxe n'est qu'apparent car il ne tient pas compte de l'intensité de l'aide ${ }^{7}$, très variable d'un pays à l'autre.

Le questionnaire Share permet d'identifier, uniquement parmi les noncohabitants, les enfants apportant une aide « quotidienne », « hebdomadaire », « mensuelle », ou « moins souvent » (graphique 4). Il est aussi demandé aux aidants d'évaluer le nombre d'heures d'aide qu'ils apportent à leur parent âgé (graphique 5, page 40). Nous rappelons que nous ne tenons pas compte ici de l'ensemble du temps passé par un individu avec son parent âgé, mais uniquement du temps qu'il consacre à apporter une aide effective (aide dans les soins personnels, aide ménagère et aide administrative) ${ }^{8}$.

\section{Graphique 4. Fréquence de l'aide apportée par les enfants aidants (cohabitants exclus)}

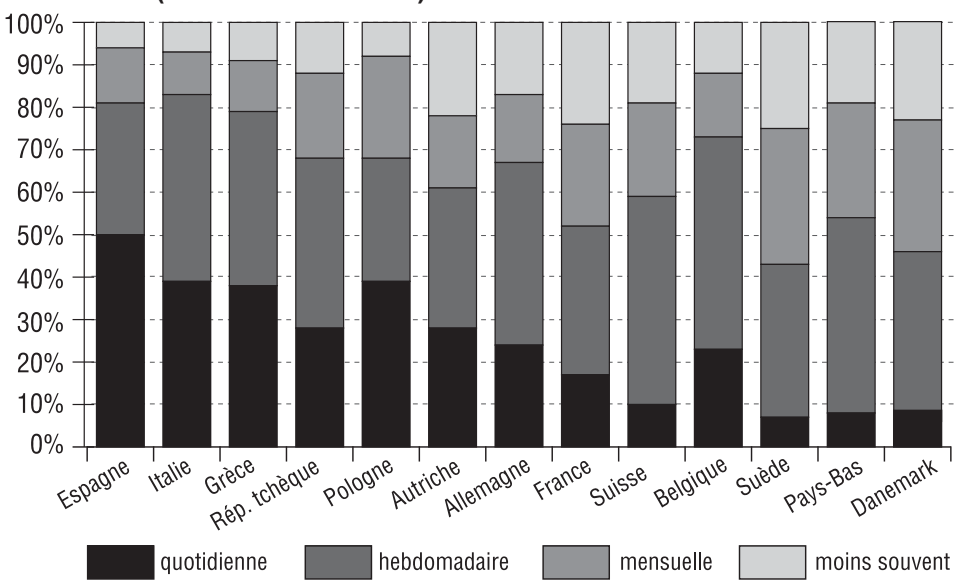

Lecture : en Espagne, 50 \% des enfants aidants hors ménage déclarent aider quotidiennement leur parent âgé, 31 \% l'aider de manière hebdomadaire, $13 \%$ de manière mensuelle et $6 \%$ moins souvent.

Champ : individus aidants hors ménage, de 50 à 65 ans, ayant un seul parent en vie.

Source : enquête Share, vague 2 (2006-2007).

7 L'intensité de l'aide fait ici référence à l'implication effective dans la prise en charge. Elle est donc appréhendée de manière restrictive car elle ne mesure pas la «charge mentale ", plus ou moins importante parmi ceux impliqués dans la prise en charge d'un parent âgé.

8 II convient par ailleurs de souligner qu'il est demandé aux individus de déclarer le nombre d'heures d'aide qu'ils apportent au cours d'une journée «normale» (s'ils aident quotidiennement), d'une semaine «normale» (s'ils aident de manière hebdomadaire), d'un mois «normal » (s'ils aident une fois par mois) ou durant les douze derniers mois (s'ils aident moins d'une fois par mois). L'information dont nous disposons ne tient donc pas compte de la variabilité et de l'évolution de l'implication durant une période donnée. La prise en charge d'un parent âgé n'est en effet pas une situation stable: entre différentes périodes de relative stabilité s'insèrent généralement des périodes de «crise» nécessitant une implication accrue de la part de l'aidant. 
La prise en compte de l'intensité de l'aide fait alors réapparaître de manière évidente le traditionnel gradient nord-sud. L'aide apportée par les seniors à leur parent âgé serait nettement plus intensive en Europe du Sud qu'en Europe du Nord. Les pays d'Europe continentale se situent encore une fois dans une situation intermédiaire. Dans le sud de l'Europe, entre 4 et 5 aidants sur 10 apportent une aide quotidienne alors qu'ils sont moins de 1 sur 10 dans ce cas dans les pays du nord de I'Europe. En Suède et au Danemark, I'aide apportée par les enfants non-cohabitants est majoritairement mensuelle ou moins fréquente. De ce point de vue, la France se place à proximité des pays d'Europe du Nord, puisque près d'un enfant aidant sur deux apporte une aide mensuelle ou moins fréquente.

La situation demeure la même si I'on considère le temps moyen passé dans I'aide plutôt que sa fréquence. Celle-ci occuperait en moyenne deux heures par jour en Espagne et une heure par jour en Italie et Grèce. Elle correspondrait à moins de deux heures par semaine aux Pays-Bas et une heure par semaine en Suède et au Danemark. Ici aussi, la situation de la France est nettement plus proche de celle des pays d'Europe du Nord puisque l'aide apportée serait légèrement inférieure à deux heures par semaine (un quart d'heure par jour).

Les pays d'Europe de l'Est, que ce soit au regard de la fréquence de l'aide ou du temps passé à aider, se positionnent sur le gradient nord-sud entre les pays du Sud et ceux d'Europe continentale.

\section{Graphique 5. Nombre d'heures d'aide apportées en moyenne par jour parmi les aidants (cohabitants exclus)}

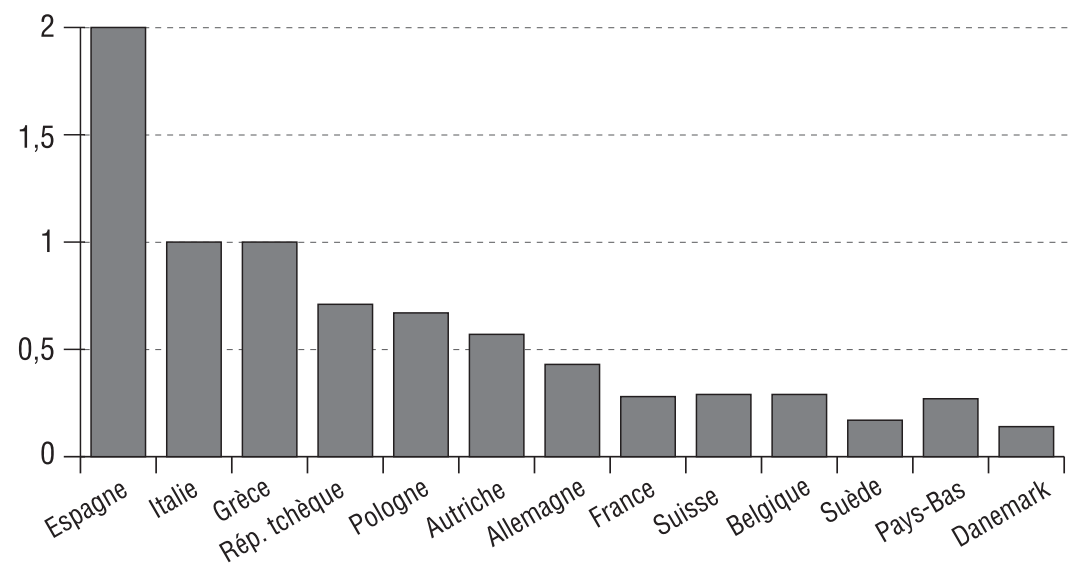

Lecture : en Espagne, les aidants consacrent en moyenne deux heures par jour à aider leur parent âgé. Champ : individus aidants hors ménage, âgés de 50 à 65 ans ayant un seul parent en vie. Source : enquête Share, vague 2 (2006-2007). 
Le comportement d'aide des seniors à leur parent serait donc au final plus hétérogène au sud qu'au nord de l'Europe. C'est en effet en Europe du Sud que l'on trouve d'une part la plus forte proportion de seniors n'aidant pas leur parent âgé et d'autre part la plus forte proportion d'aidants « intensifs ». Ce résultat peut s'expliquer de différentes manières. II peut tout d'abord révéler les différences institutionnelles qui existent entre pays. Il est par exemple possible que les parents présentant des incapacités sévères soient plus fréquemment en institution dans le nord de l'Europe que dans le sud, si bien que la nécessité d'apporter une aide " intensive » est plus importante au sud qu'au nord. Il peut aussi s'expliquer par un biais de déclaration entre pays européens. Il est par exemple possible que dans le sud de l'Europe les individus apportant une aide occasionnelle à leur parent se déclarent moins fréquemment aidants que dans le nord. Enfin, nous ne tenons pas compte ici de I'aide potentielle que peuvent fournir les frères et sœurs des seniors considérés, voire d'autres membres de la famille ou de l'entourage. Ce résultat pourrait alors s'expliquer par le fait que la production d'aide autour des personnes âgées est plus fréquemment portée par un aidant unique dans le sud de l'Europe alors qu'elle peut être plus souvent partagée dans le nord de l'Europe.

\section{Une aide en majorité apportée par les filles à leur mère}

Parmi les individus dont l'un des deux parents est décédé, il s'agit dans près de 9 cas sur 10 du père. L'aide que les seniors apportent à leur parent est donc dans notre échantillon très majoritairement une aide destinée à leur mère (dans $88 \%$ des cas).

Par ailleurs, dans $60 \%$ des cas l'enfant aidant est une fille, confirmant, quel que soit le pays considéré, que les femmes sont plus fréquemment impliquées dans l'aide informelle que les hommes (tableau 2, page 42). $C^{\prime}$ est en particulier en Autriche que I'on retrouve le plus de femmes parmi les aidants, alors que $c^{\prime}$ est dans le nord que la répartition par sexe des aidants est la moins inégalitaire. Le tableau 3, page 42 montre par ailleurs que la différence homme femme s'accentue à mesure que I'intensité de l'aide augmente. Parmi la majorité des aidants, c'est-à-dire ceux apportant moins d'une heure d'aide par jour à leur parent âgé, un peu moins de 6 sur 10 sont des femmes. Elles représentent en revanche plus de 7 aidants sur 10 quand I'aide dépasse deux heures par jour.

Le tableau 4, page 43 présente par pays le temps moyen que consacrent les aidants hors ménage dans l'aide à leur parent. Les femmes s'impliqueraient en moyenne deux fois plus que les hommes ( 3 heures par semaine pour les femmes contre 1 heure 15 par semaine pour les 
hommes). Mis à part les Pays-Bas, tous les pays sont caractérisés par cette plus forte implication des femmes. À noter enfin que parmi les pays d'Europe continentale, la différence homme femme apparaît plus faible en France que chez ses voisins allemands, autrichiens, belges et suisses.

Au final, I'aide des seniors à leur parent est dans $52 \%$ des cas l'aide $\mathrm{d}^{\prime}$ une fille à sa mère ${ }^{9}$, la proportion passant à $62 \%$ lorsque l'on restreint les aidants aux non-cohabitants apportant en moyenne plus d'une heure d'aide par jour.

\section{Tableau 2. Proportion de femmes parmi les seniors apportant une aide à leur parent}

\begin{tabular}{|c|c|c|c|c|c|c|c|}
\hline \multicolumn{2}{|c|}{ Europe du Sud } & \multicolumn{2}{|c|}{ Europe de l'Est } & \multicolumn{2}{|c|}{ Europe continentale } & \multicolumn{2}{|c|}{ Europe du Nord } \\
\hline \multicolumn{2}{|c|}{$62 \%$} & \multicolumn{2}{|l|}{$64 \%$} & \multicolumn{2}{|c|}{$60 \%$} & \multicolumn{2}{|c|}{$57 \%$} \\
\hline Espagne & $59 \%$ & République tchèque & $67 \%$ & Autriche & $69 \%$ & Suède & $55 \%$ \\
\hline Italie & $63 \%$ & Pologne & $60 \%$ & Allemagne & $59 \%$ & Pays-Bas & $61 \%$ \\
\hline \multirow[t]{3}{*}{ Grèce } & $61 \%$ & & & France & $58 \%$ & Danemark & $55 \%$ \\
\hline & & & & Suisse & $60 \%$ & & \\
\hline & & & & Belgique & $62 \%$ & & \\
\hline \multicolumn{8}{|c|}{ Ensemble : 60 \% } \\
\hline
\end{tabular}

Lecture: en Espagne, 59 \% des enfants aidants sont des femmes.

Champ: individus aidants (hors ménage ou cohabitants), de 50 à 65 ans ayant un seul parent en vie.

Source: enquête Share, vague 2 (2006-2007).

\section{Tableau 3. Distribution des aidants selon le nombre d'heures d'aide apportées par jour (cohabitants exclus)}

\begin{tabular}{l|c|c|c} 
& \multicolumn{3}{c}{ Proportion d'aidants « hors ménage » } \\
& Proportion totale & Femmes & Hommes \\
\hline moins d'1 heure & $70 \%$ & $57 \%$ & $43 \%$ \\
\hline entre 1 heure et 2 heures & $13 \%$ & $64 \%$ & $36 \%$ \\
\hline entre 2 heures et 4 heures & $11 \%$ & $76 \%$ & $24 \%$ \\
\hline entre 4 heures et 8 heures & $5 \%$ & $79 \%$ & $21 \%$ \\
\hline plus de 8 heures & $2 \%$ & $73 \%$ & $27 \%$ \\
\hline
\end{tabular}

Lecture: dans l'ensemble de l'échantillon, 70 \% des aidants " hors ménage " en Europe apportent moins d'une heure d'aide par jour. Parmi eux, $57 \%$ sont des femmes.

Champ: individus aidants " hors ménage ", de 50 à 65 ans ayant un seul parent en vie.

Source: enquête Share, vague 2 (2006-2007).

9 Dans $35 \%$ des cas, l'aide est apportée par un fils à sa mère, dans $7 \%$ des cas par une fille à son père, et enfin dans $5 \%$ des cas par un fils à son père. 
Tableau 4. Temps moyen consacré par les seniors aidants dans l'aide à leur parent (cohabitants exclus)

\begin{tabular}{|c|c|c|c|c|} 
& \multicolumn{2}{c}{ Par jour } & \multicolumn{2}{c}{ Par semaine } \\
\cline { 2 - 5 } & Hommes & Femmes & Hommes & Femmes \\
\hline Europe du Sud & $\mathbf{0 , 7 6}$ & $\mathbf{1 , 3 5}$ & $\mathbf{5 , 3 2}$ & $\mathbf{9 , 4 3}$ \\
\hline Espagne & 1,42 & 2,00 & 9,94 & 14,00 \\
\hline Italie & 0,71 & 1,14 & 4,97 & 7,98 \\
\hline Grèce & 0,57 & 1,34 & 3,99 & 9,38 \\
\hline Europe de l'Est & $\mathbf{0 , 6 6}$ & $\mathbf{0 , 9 3}$ & $\mathbf{4 , 5 9}$ & $\mathbf{6 , 5 2}$ \\
\hline République tchèque & 0,64 & 0,83 & 4,48 & 5,81 \\
\hline Pologne & 0,69 & 1,14 & 4,83 & 7,98 \\
\hline Europe continentale & $\mathbf{0 , 2 1}$ & $\mathbf{0 , 3 8}$ & $\mathbf{1 , 4 5}$ & $\mathbf{2 , 6 7}$ \\
\hline Autriche & 0,11 & 0,57 & 0,77 & 3,99 \\
\hline Allemagne & 0,29 & 0,43 & 2,03 & 3,01 \\
\hline France & 0,27 & 0,34 & 1,89 & 2,38 \\
\hline Suisse & 0,14 & 0,34 & 0,98 & 2,38 \\
\hline Belgique & 0,14 & 0,36 & 0,98 & 2,52 \\
\hline Europe du Nord & $\mathbf{0 , 1 2}$ & $\mathbf{0 , 1 8}$ & $\mathbf{0 , 8 2}$ & $\mathbf{1 , 2 8}$ \\
\hline Suède & 0,14 & 0,23 & 0,98 & 1,61 \\
\hline Pays-Bas & 0,14 & 0,14 & 0,98 & 0,98 \\
\hline Danemark & 0,07 & 0,17 & 0,49 & 1,19 \\
\hline Ensemble & $\mathbf{0 , 1 7}$ & $\mathbf{0 , 4 3}$ & $\mathbf{1 , 1 9}$ & $\mathbf{3 , 0 1}$ \\
\hline
\end{tabular}

Lecture: en Espagne, les hommes aidants hors ménage consacrent en moyenne 1,42 heure ( 1 heure et 25 minutes) par jour à l'aide de leur parent, soit 9,94 heures ( 9 heures et 56 minutes) par semaine.

Champ: individus aidants " hors ménage ", de 50 à 65 ans, ayant un seul parent en vie.

Source: enquête Share, vague 2 (2006-2007).

\section{L'état de santé des aidants}

Certaines études mettent en évidence le fait que l'aide apportée aux personnes âgées dépendantes aurait des effets négatifs sur l'état de santé des aidants (Dutheil, 2002; Fondation Médéric Alzheimer, 2001). La relation entre aide et état de santé est importante au regard de l'analyse que nous souhaitons mener car elle peut agir, comme nous le verrons par la suite, comme un facteur confondant dans l'étude de l'arbitrage entre aide et emploi. Cette relation est cependant complexe et l'effet de I'aide sur la santé de l'aidant difficile à identifier.

Tout d'abord, l'état de santé joue sur la capacité qu'ont les individus à venir en aide à un proche dépendant. Cela tend à réduire la relation négative pouvant exister entre aide et état de santé. Les individus déclarant un mauvais état de santé ont en particulier plus de chance d'appartenir à la population des non-aidants qu'à celle des aidants. Le graphique 6 (page 44) semble illustrer ce phénomène. Indépendamment de l'intensité de l'aide, les individus apportant un soutien à leur parent 
apparaissent en effet moins nombreux que les non-aidants à déclarer au cours de l'enquête un état de santé « médiocre » ou « acceptable » ${ }^{\mathbf{1 0}}$.

Deuxièmement, l'état de santé des enfants est lié à l'état de santé des parents (Ahlburg, 1998; Case, 2002; Devaux, 2008). Cela tend à accentuer la relation négative pouvant exister entre aide et état de santé. Il est en effet possible que les individus caractérisés par un mauvais état de santé aient, toutes choses égales par ailleurs, une plus forte probabilité d'avoir un parent dont l'état de santé nécessite une prise en charge. Les aidants tendraient donc à avoir un moins bon état de santé que les non-aidants sans pour autant que I'on puisse conclure à un effet négatif de l'aide sur l'état de santé des aidants.

Le graphique 7 (page 45) montre que plus le temps passé dans l'aide augmente, plus l'état de santé des aidants se détériore. Les seniors aidant " à temps plein », c'est-à-dire ceux aidant en moyenne plus de huit heures par jour leur parent, sont $50 \%$ à déclarer un état de santé «médiocre » ou " acceptable » alors qu'ils sont moins de $20 \%$ dans ce cas parmi ceux apportant en moyenne moins d'une heure d'aide par jour. On ne peut cependant pas savoir s'il s'agit d'un effet négatif de l'aide sur l'état de santé ou d'un effet de sélection lié à la corrélation entre l'état de santé des enfants et l'état de santé des parents.

\section{Graphique 6. État de santé déclaré par les aidants et les non-aidants}

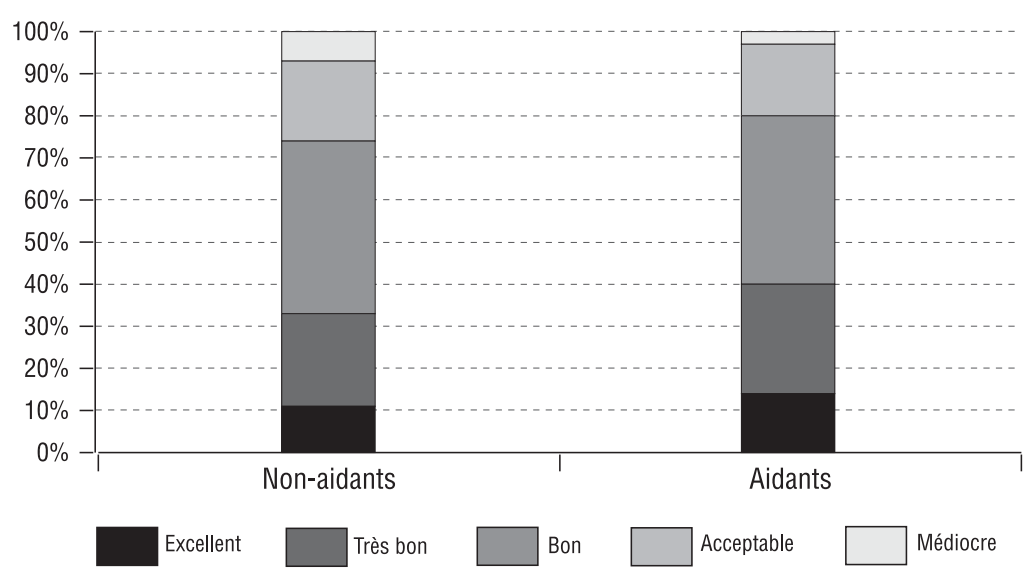

Lecture : dans l'ensemble de l'échantillon les aidants sont $11 \%$ à déclarer un état de santé " excellent ", $22 \%$ un état de santé " très bon », $41 \%$ un état de santé " bon », $19 \%$ un état de santé « acceptable » et $7 \%$ un état de santé « médiocre ".

Champ : individus de 50 à 65 ans ayant un seul parent en vie.

Source : enquête Share, vague 2 (2006-2007).

10 «Médiocre » et «acceptable » sont les traductions dans le cas français de «fair » et «poor» employés dans la version générique (en anglais) du questionnaire. 


\section{Graphique 7. État de santé déclaré par les aidants selon le temps passé à aider en moyenne par jour (cohabitants exclus)}

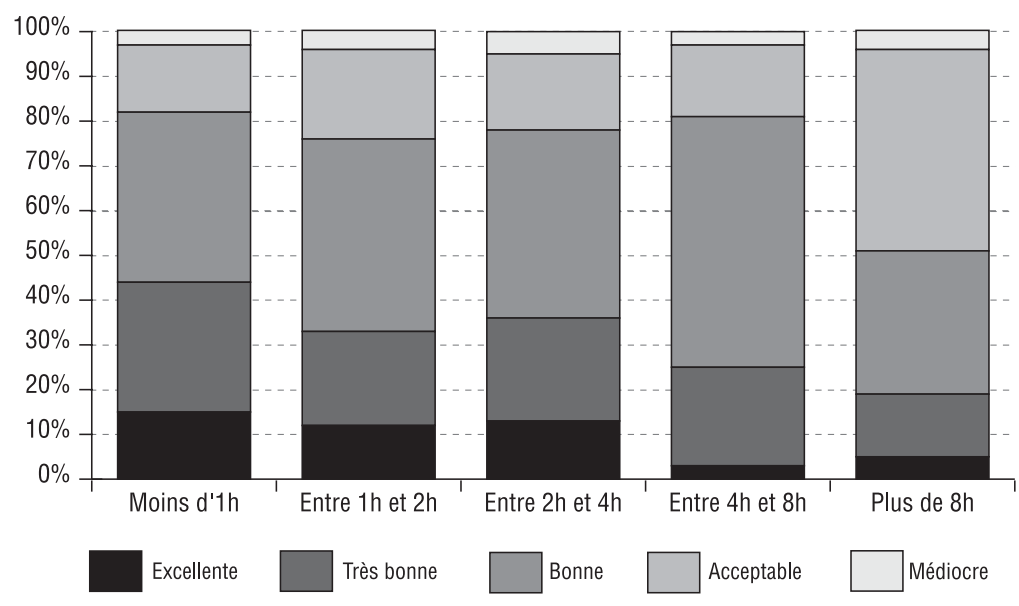

Lecture : dans l'ensemble de l'échantillon, les individus apportant moins d'une heure d'aide par jour sont $15 \%$ à déclarer un état de santé « excellent ", $29 \%$ un état de santé " très bon ", $38 \%$ un état de santé " bon », $15 \%$ un état de santé " acceptable » et $2 \%$ un état de santé " médiocre ».

Champ : individus aidants « hors ménage " de 50 à 65 ans ayant un seul parent en vie.

Source : enquête Share, vague 2 (2006-2007).

\section{Activité professionnelle des aidants}

En plus de leur potentielle activité d'aidant, les seniors peuvent exercer, pour une majorité d'entre eux, une activité professionnelle rémunérée. L'objectif est ici d'étudier la manière dont les individus articulent ces deux activités. L'aide à un proche peut en effet contraindre plus ou moins fortement l'exercice d'une activité professionnelle. Au regard de la population ici étudiée, la question du renoncement à l'exercice d'une activité professionnelle revêt un sens particulier car la question d'aider ou non un parent âgé peut interagir avec celle du départ à la retraite. Le fait d'aider un parent âgé peut en effet inciter les seniors à quitter prématurément le marché du travail ou freiner leur souhait de prolonger leur activité professionnelle au-delà de l'âge légal de la retraite.

\section{Les différents effets de l'aide sur l'emploi}

La prise en charge d'un parent âgé dépendant peut a priori avoir plusieurs effets sur l'offre de travail des aidants. Trois effets sont mis en avant dans la littérature économique: un " effet de substitution », un " effet revenu » et un « effet répit » (Carmichael et Charles, 1998). Le premier tend à diminuer l'offre de travail alors que les deuxième et 
troisième tendent au contraire à l'augmenter. Au final, I'effet global demeure donc a priori indéterminé.

Le principal effet auquel on peut s'attendre est négatif: aider se traduit par une diminution de l'offre de travail. II est dû au fait que les individus doivent en partie arbitrer entre temps consacré à travailler et temps consacré à aider. La substitution entre temps d'aide et temps de travail n'est cependant pas automatique, les individus ayant aussi la possibilité de renoncer à du loisir pour venir en aide à un proche dépendant ${ }^{11}$. L'enquête qualitative effectuée en France par Le Bihan et Martin (2006) auprès de femmes apportant une aide à leur parent âgé dépendant irait assez largement dans ce sens. Les enquêtées mettent en particulier en avant I'importance qu'elles accordent à leur activité professionnelle et le souhait de ne pas recourir à des aménagements de leur vie professionnelle (réduction du temps de travail, préretraite). En revanche, elles évoquent le fait que l'aide apportée à leur parent se fait au détriment de leur vie familiale et personnelle.

À côté de cet effet potentiellement négatif de I'aide sur I'emploi, la littérature économique évoque deux effets agissant à contresens, incitant les aidants à offrir plus de travail que les non-aidants (Carmichael et Charles, 1998). Le premier est qualifié $d^{\prime}$ " effet revenu ». Il correspond au fait que la prise en charge d'un parent âgé dépendant peut s'avérer coûteuse, ce qui peut inciter les aidants à augmenter leur offre de travail afin d'accroître leurs ressources. Le deuxième effet positif est $I^{\prime}$ « effet répit ». II illustre le fait que certains individus fortement impliqués dans la prise en charge d'un proche peuvent ressentir le besoin d'exercer une activité professionnelle afin de se libérer de leur rôle d'aidant.

Il semble par ailleurs que l'exercice d'une activité professionnelle agisse comme un rempart permettant aux individus, principalement les femmes, de ne pas «basculer totalement dans la fonction d'aide » (Le Bihan et Martin, 2006). Cet effet, comme nous le verrons par la suite, pourrait expliquer pourquoi les individus apportant une aide inférieure à une heure par jour occupent plus fréquemment une activité professionnelle que les non aidants.

\section{Les effets mis en évidence dans la littérature empirique}

L'effet global étant a priori indéterminé, un certain nombre d'études économiques visent à tester empiriquement l'effet de l'aide sur l'offre de travail.

11 Le terme de loisir est ici employé dans sa définition économique. II caractérise tout le temps non consacré à du travail rémunéré. 
En Europe ${ }^{12}$, les premières études sur le sujet ont été effectuées sur données anglaises. Sur un échantillon constitué uniquement de femmes âgées de 21 à 59 ans, Carmichael et Charles (1998) montrent que le fait d'apporter une aide à un proche aurait un effet positif sur la participation au marché du travail pour celles aidant moins de vingt heures par semaine, tandis que cela aurait un effet négatif pour celles aidant plus de vingt heures par semaine. Une étude plus récente, toujours sur données anglaises, montre par ailleurs que l'effet négatif de l'aide au-delà d'un certain seuil serait plus faible pour les hommes que pour les femmes, et que la cohabitation serait plus néfaste à l'emploi que l'aide « hors ménage » (Carmichael et Charles, 2003). L'étude de Heitmueller (2007), sur données plus récentes, confirme ces résultats dans le cas anglais: I'aide à "distance » au-delà de vingt heures par semaine et la cohabitation avec un parent âgé auraient un effet négatif sur l'emploi.

En dehors de I'Angleterre, seules trois études à notre connaissance analysent I'effet de I'aide à un proche sur l'offre de travail. Crespo (2007), utilisant les données de la première vague de l'enquête Share, montre que dans les pays d'Europe du Nord et d'Europe du Sud, I'aide quotidienne apportée par les femmes réduirait leur participation au marché du travail ${ }^{13}$. L'étude de Bolin et al. (2008), utilisant elle aussi les données de la première vague de l'enquête Share, mais à travers une méthode économétrique différente, montre que le fait d'aider un parent âgé aurait un effet négatif sur la participation au marché du travail uniquement pour les hommes des pays d'Europe continentale. Enfin, Casado-Marín et al. (2008) distinguent dans leurs estimations les femmes qui travaillaient avant d'aider de celles qui ne travaillaient pas. Ils montrent alors que parmi les femmes exerçant une activité professionnelle, celles devenant aidantes ne renoncent pas à leur emploi, quel que soit le groupe de pays considéré. En revanche, dans les pays d'Europe du Sud et d'Europe continentale, les femmes n'exerçant pas d'activité professionnelle au moment de devenir aidantes participent par la suite moins fréquemment que les autres au marché du travail. Toutes ces études mettent donc en lumière une relation entre aide et emploi, même si les résultats divergent quant aux modalités de cette relation et aux pays concernés.

\section{Les aidants occupent en moyenne plus souvent une activité professionnelle que les non-aidants}

Une première approche de la relation entre aide et emploi à travers les données de la deuxième vague de Share montre que le fait d'être impliqué

12 cf. Ettner (1995, 1996), Johson et Lo Sasso (2000), Pezzin et Schone (1999), Wolf et Soldo (1994) pour des travaux sur données américaines. Pour une étude récente sur données australiennes, se reporter à Berecki-Gisolf et al. (2008).

13 L'auteur ne teste pas l'effet sur les pays d'Europe continentale. 
ou non dans la prise en charge d'un parent âgé ne semble pas avoir d'effet évident sur l'offre de travail. Pris dans leur globalité, c'est-à-dire indépendamment de l'intensité de leur implication, les aidants ne travaillent pas moins que les non aidants. Au contraire, les aidants seraient légèrement plus nombreux à exercer une activité professionnelle (53\% contre $50 \%$ ), et ce bien que les femmes, moins souvent actives que les hommes, soient surreprésentées dans la population des aidants. Ce résultat est semblable à celui obtenu par Carmichael et Charles (1998) sur données anglaises. Cette légère surreprésentation des actifs occupés dans la population des aidants ne peut s'expliquer par une différence d'âge entre les deux populations, l'âge moyen étant quasiment identique dans les deux populations ${ }^{14}$.

Ce résultat peut en revanche rendre compte du fait que la majorité des aidants $(70 \%)$ apportent moins d'une heure d'aide par jour à leur parent âgé, ce qui limite a priori les effets négatifs de l'aide sur l'exercice d'une activité professionnelle. II convient donc de distinguer l'activité des aidants suivant le nombre d'heures d'aide qu'ils apportent à leur parent âgé.

\section{Une relation décroissante entre nombre d'heures d'aide et taux d'emploi}

Il apparaît alors que le taux d'emploi des aidants varie considérablement suivant I'intensité de l'aide qu'ils fournissent (graphique 8, page 49). II décroît de manière continue avec le nombre d'heures d'aide apportée au parent âgé ${ }^{15}$. Il passe de $63 \%$ pour ceux apportant en moyenne moins d'une heure d'aide par jour à $5 \%$ pour ceux apportant plus de huit heures d'aide par jour. Ce résultat peut révéler un effet négatif de I'aide sur l'emploi, effet croissant avec l'intensité de l'aide.

Nous verrons par la suite que d'autres interprétations sont envisageables pour expliquer la corrélation négative entre le nombre d'heures d'aide et la proportion d'individus exerçant une activité professionnelle.

Mais alors que les aidants "intensifs ", c'est-à-dire ceux qui apportent plus d'une heure d'aide par jour, exercent moins souvent une activité professionnelle que les non-aidants, les aidants " non intensifs », c'està-dire ceux qui apportent moins d'une heure d'aide par jour, occupent en moyenne plus fréquemment une activité professionnelle que les non-

14 Parmi les femmes, les aidantes ont en moyenne 55,3 ans, contre 55,1 ans en moyenne pour les non-aidantes. Parmi les hommes, les aidants ont en moyenne 55,7 ans, et les non-aidants en moyenne 55,5 ans. L'âge n'est donc pas ici un facteur pouvant expliquer des différences entre l'activité moyenne des deux populations.

15 Nous rappelons que la prise en compte de l'intensité de l'aide nous contraint à exclure de l'analyse les enfants cohabitant avec leur parent. 
aidants. Ce résultat peut simplement rendre compte du fait que les individus aidant leur parent tout en exerçant une activité professionnelle apportent en majorité une aide inférieure à une heure par jour. II peut aussi s'expliquer par l'effet mis en évidence par Le Bihan et Martin (2006) : les aidants « non intensifs » pourraient plus fréquemment exercer une activité professionnelle pour se protéger d'une implication plus importante dans l'activité $d^{\prime}$ « aidant ».

La relation entre aide et emploi est sans doute fonction du contexte institutionnel et culturel dans lequel elle se situe et I'on peut dès lors s'attendre à ce qu'elle varie d'un pays à l'autre. Parmi les données dont nous disposons, les effectifs d'aidants, ceux aidant de manière " intensive » en particulier, sont cependant trop faibles au sein de chaque pays pour permettre une comparaison statistique raisonnable. Nous proposons donc une comparaison par groupe de pays. Même si cette comparaison peut noyer certaines différences entre pays d'un même groupe, elle semble justifiée au regard de l'analyse effectuée précédemment sur les comportements d'aide.

La tendance selon laquelle les aidants « non intensifs» sont plus souvent actifs occupés que les non-aidants, alors que les aidants « intensifs »le seraient moins souvent, est commune à tous les groupes de pays et concerne aussi bien les hommes que les femmes (tableau 5, page 50). Seuls les hommes en Europe de l'Est échappent à la règle: même aidants " intensifs», ils seraient plus souvent actifs occupés que les non-aidants. La différence de taux d'emploi entre non-aidants et aidants "intensifs » est globalement plus importante pour les femmes que pour les hommes. En Europe du Sud en particulier, les femmes apportant plus d'une heure d'aide par jour seraient moins de 2 sur 10 à exercer une activité professionnelle, soit près de la moitié moins que les non aidantes.

\section{Graphique 8. Taux d'emploi selon le comportement d'aide (cohabitants exclus)}

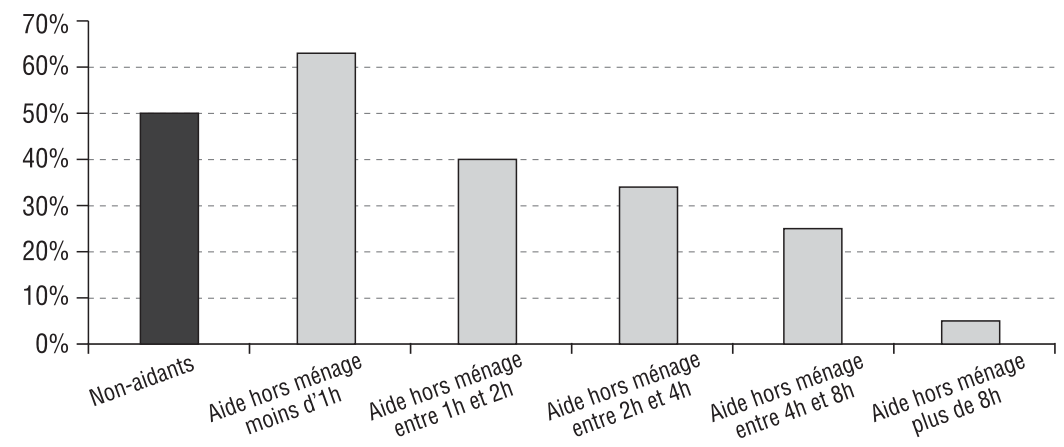

Lecture : dans l'ensemble de l'échantillon, $50 \%$ des individus non-aidants exercent une activité professionnelle.

Champ : individus de 50 à 65 ans ayant un seul parent en vie.

Source : enquête Share, vague 2 (2006-2007). 
Tableau 5. Taux d'emploi des seniors selon le comportement d'aide (cohabitants exclus)

\begin{tabular}{|c|c|c|c|c|}
\hline & Europe du Nord & Europe continentale & Europe du Sud & Europe de l'Est \\
\hline Non-aidantes & $58 \%(361)$ & $45 \%(597)$ & $30 \%(400)$ & $31 \%(278)$ \\
\hline Aidantes hors ménage & $62 \%(283)$ & $47 \%(335)$ & $23 \%(133)$ & $31 \%(100)$ \\
\hline $\begin{array}{c}\text { moins d'1h par jour } \\
\text { 1h par jour ou plus }\end{array}$ & $67 \%(239)$ & $54 \%(210)$ & $35 \%(40)$ & $40 \%(45)$ \\
\hline & $34 \%(44)$ & $35 \%(125)$ & $17 \%(93)$ & $24 \%(55)$ \\
\hline Non-aidants & Europe du Nord & Europe continentale & Europe du Sud & Europe de l'Est \\
\hline Aidants hors ménage & $70 \%(358)$ & $55 \%(558)$ & $61 \%(423)$ & $47 \%(239)$ \\
\hline moins d'1h par jour & $74 \%(211)$ & $63 \%(209)$ & $53 \%(74)$ & $73 \%(49)$ \\
\hline 1h par jour ou plus & $65 \%(20)$ & $68 \%(149)$ & $63 \%(38)$ & $78 \%(32)$ \\
\hline
\end{tabular}

Lecture : 52 \% des femmes non-aidantes en Europe du Nord exercent une activité professionnelle.

Note : entre parenthèses figurent les effectifs.

Champ : individus de 50 à 65 ans ayant un seul parent en vie.

Source : enquête Share, vague 2 (2006-2007).

\section{Aider un parent âgé se fait-il au détriment de l'emploi?}

En comparant l'activité des aidants avec celle des non-aidants, nous avons jusque-là implicitement appréhendé I'aide comme un déterminant de I'offre de travail. Sous cette hypothèse, la corrélation négative qui existe entre le nombre d'heures d'aide apportées et le fait d'exercer ou non une activité professionnelle reflète un effet négatif de l'aide sur l'emploi.

Cependant, I'analyse statistique faite précédemment souffre potentiellement de deux biais qu'il convient de contrôler par une procédure économétrique. Le premier correspond à un «biais de variables omises » et le second à un « un biais de simultanéité ».

Tout d'abord, un certain nombre de caractéristiques individuelles peuvent, si elles ne sont pas considérées lors de l'analyse, conduire à une corrélation entre aide et emploi alors même que les décisions de venir en aide à un parent et d'exercer une activité professionnelle sont indépendantes. Le fait d'étudier la relation entre aide et emploi sans tenir compte de l'état de santé des individus peut par exemple biaiser l'analyse. Si par exemple un bon état de santé favorise à la fois l'exercice d'une activité professionnelle et la fourniture d'aide, la non-prise en compte de cette variable peut conduire à une corrélation positive entre aide et emploi, qui pourra à tort 
être interprétée comme un effet positif de I'aide sur l'emploi. Pour contrôler l'effet confondant d'un certain nombre de caractéristiques individuelles telles que l'état de santé, nous avons effectué une estimation économétrique mesurant l'effet de l'aide apportée à un parent âgé sur la probabilité d'exercer une activité professionnelle, toutes choses égales par ailleurs. L'estimation que nous proposons permet en outre de tenir compte de l'existence d'un autre biais.

II est en effet possible que l'activité soit un déterminant de l'aide et non l'inverse. La corrélation négative entre quantité d'aides et probabilité d'exercer une activité professionnelle peut alors s'expliquer par le fait que l'inactivité favorise la fourniture d'aide informelle, les inactifs ayant des coûts d'opportunité généralement plus faibles que les actifs. Les individus ayant pris leur retraite avant que se pose la question de l'aide à un parent âgé ne sont par exemple pas concernés par l'arbitrage entre aide et emploi. Qu'ils aident ou non n'a au final aucune conséquence sur leur offre de travail, qui sera nulle quel que soit leur comportement d'aide. Cependant, le fait que les inactifs aident plus que les actifs occupés peut brouiller l'analyse car cela conduit à une corrélation négative entre aide et travail, pouvant être interprétée à tort comme un effet négatif de I'aide sur l'offre de travail. Afin de contrôler la possible endogénéité de I'aide par rapport à la position vis-à-vis du marché du travail, nous avons estimé conjointement la décision d'aider et la décision d'exercer ou non une activité professionnelle (encadré 1).

\section{- EnCADRÉ 1}

\section{Traitement de l'endogénéité de l'aide}

Afin d'évaluer l'effet de l'aide sur l'emploi, il convient de contrôler la potentielle endogénéité de l'aide, provenant du fait que l'aide peut être expliquée par l'offre de travail et non l'inverse.

À l'instar de Casado-Marín et al. (2008) et Berecki-Gisolf et al. (2008), il est possible d'utiliser des données longitudinales afin d'identifier les conséquences de l'arbitrage entre aide et emploi uniquement sur une population pour qui l'arbitrage existe. La méthode consiste à tester économétriquement en vague 2 l'effet de l'aide sur la probabilité de travailler uniquement parmi les individus exerçant une activité professionnelle en vague 1. En excluant de l'analyse les individus qui en vague 1 sont sortis du marché du travail, on exclut par la même occasion ceux pour qui l'arbitrage n'existe pas en vague 2. La dimension longitudinale de l'enquête Share nous permet d'appliquer cette méthodologie. Elle n'est cependant pas utilisée ici car elle ne fait que réduire le problème d'endogénéité sans véritablement le régler, la relation de causalité allant de la position vis-à-vis du marché du travail à la décision d'aide n'étant pas totalement contrôlée. Elle n'élimine en effet pas tous les individus pour qui l'arbitrage entre aide et emploi n'existe pas ${ }^{16}$.

16 II est possible que les individus sortis du marché du travail entre les deux vagues soient, justement à cause de leur inactivité, plus propices à venir en aide à un proche dépendant. Pour eux, la causalité va donc bien de la position vis-à-vis du marché au comportement d'aide et non l'inverse. Cette méthode ne permet donc par réellement de gérer le «biais de simultanéité ». 
Une autre méthode consiste à tester économétriquement l'effet de l'aide sur l'offre de travail en instrumentant le comportement d'aide (Bolin et al., 2008 ; Ettner, 1995, 1996 ; Heitmueller, 2007). L'objectif est alors de considérer dans le comportement d'aide uniquement ce qui ne peut pas être expliqué par le comportement d'offre de travail. Le niveau d'incapacité du parent pourrait par exemple être qualifié de bon instrument car il explique le comportement d'aide, mais est a priori indépendant de l'offre de travail à comportement d'aide donné. Dans ce cas, si le niveau d'incapacité influence significativement l'offre de travail, toutes choses égales par ailleurs, c'est que le comportement d'aide a un effet direct sur l'offre de travail. Cette méthode est a priori la plus adaptée mais elle bute sur la difficulté que peut avoir l'économètre à disposer de bons instruments. L'enquête Share offre de ce point de vue un nombre limité d'instruments. Ceux utilisés par Bolin et al. (2008), à partir de la première vague de l'enquête Share, ont par exemple un faible pouvoir explicatif de l'aide et peuvent pour certains être fortement suspectés d'endogénéité (comme la distance géographique séparant le senior de son parent).

La méthode que nous proposons ici consiste à estimer un probit bivarié permettant de modéliser de manière jointe l'offre de travail et l'offre d'aide. Chacune des deux variables est caractérisée de manière dichotomique. La première équation modélise l'effet de la participation au marché du travail (être ou non actif occupé) et d'autres variables explicatives (âge et état de santé du parent, sexe de l'enfant, etc.) sur le fait d'apporter ou non une aide à son parent âgé. La seconde équation mesure l'effet du comportement d'aide (apporter ou non une aide à son parent âgé) et d'autres variables explicatives (âge de l'enfant, niveau d'éducation de l'enfant, etc.) sur le fait d'être ou non actif occupé. Ce type de modèle a précédemment été utilisé afin de modéliser le comportement de deux agents dont les décisions sont interdépendantes (Bjorn et Vuong 1985 ; Fontaine et al., 2009; Krauth, 2006; Soetevent et Kooreman, 2007). Il est ici utilisé dans un cadre différent puisque nous modélisons deux décisions prises par un agent unique. L'estimation de ce type de modèle nécessite au préalable de traiter le fait qu'ils sont incomplets (Heckman, 1978 ; Maddala, 1983 ; Tamer, 2003). La méthode utilisée ici pour gérer cette incomplétude est identique à celle utilisée par Fontaine et al. (2009), à la différence près que la règle de sélection est ici posée de manière exogène ${ }^{17}$. Nous supposons par ailleurs que les résidus des deux équations sont indépendants et suivent tous deux une loi normale.

Les résultats sont présentés dans le tableau 6, page 56. Deux estimations ont été effectuées. Dans la première (estimation 1), le comportement d'aide est appréhendé à travers une variable dichotomique valant 1 si l'individu apporte une aide hors ménage à son parent (quelle que soit l'intensité de I'aide), et 0 sinon. La relation entre aide et emploi est alors étudiée quelle que soit l'intensité de l'aide apportée. Dans la deuxième (estimation 2), le comportement d'aide est appréhendé à travers une variable dichotomique valant 1 si l'individu apporte une aide hors ménage supérieure à une heure par jour, 0 sinon. Dans ce cas, on suppose que l'arbitrage entre aide et emploi n'existe qu'au-delà d'une certaine quantité d'aide, le seuil d'une heure par jour étant suggéré par l'analyse statistique précédente. La prise en compte de l'intensité de l'aide dans la deuxième estimation contraint à I'exclusion des cas où les individus cohabitent avec leur parent. Par souci de comparaison, nous les avons aussi éliminés de l'estimation 1. Plutôt que de tester si l'effet de l'aide sur l'exercice d'une activité professionnelle 
est différent pour les femmes et les hommes, nous avons préféré tester des différences entre les groupes de pays jusque-là considérés ${ }^{18}$.

\section{Les déterminants de l'exercice d'une activité professionnelle}

Au regard du comportement face à l'emploi (colonnes 2 et 4 du tableau 6), les résultats des deux estimations sont quasiment identiques. Comparés aux Français, les Suédois et les Suisses sont les seuls à avoir, toutes choses égales par ailleurs, une plus forte probabilité d'exercer une activité professionnelle. En Autriche, Belgique, Italie et Pologne, les seniors sont en revanche, toutes choses égales par ailleurs, caractérisés par une plus faible probabilité d'être actif occupé qu'en France.

De manière prévisible, les femmes, les seniors les plus âgés ou ceux n'ayant pas fait d'études supérieures exercent moins fréquemment que les autres une activité professionnelle.

Le fait d'exercer une activité professionnelle semble par ailleurs affecté par l'état de santé des individus. Ceux déclarant un état de santé « médiocre » ou « acceptable » exercent en effet moins fréquemment que les autres une activité professionnelle. À noter que l'état de santé peut cependant souffrir d'un biais d'endogénéité car nous ne tenons pas compte ici des conséquences de l'exercice d'une activité professionnelle sur l'état de santé.

Enfin, le fait d'avoir ou non un conjoint semble n'avoir aucun effet sur l'activité professionnelle.

\section{Les déterminants de l'aide à un parent âgé}

Au regard du comportement d'aide (colonnes 1 et 3 du tableau 6), les estimations confirment en grande partie les différences entre pays observés précédemment.

Parmi les pays d'Europe continentale, l'Autriche apparaît bien, toutes choses égales par ailleurs, comme le pays où les seniors sont le moins fréquemment impliqués dans l'aide à un parent. Cette particularité semble concerner les aidants "non intensifs», les Autrichiens ne se distinguant

18 Le nombre d'individus apportant plus d'une heure d'aide par jour étant trop réduit dans l'échantillon, en particulier dans les pays d'Europe du Nord, il nous est impossible de tester simultanément si au sein de chaque groupe de pays existent des différences par sexe. 
pas des autres pays en ce qui concerne le fait d'apporter plus d'une heure d'aide par jour. La Belgique apparaît quant à elle le pays où les seniors sont le plus fréquemment impliqués dans l'aide à un parent âgé, que I'on considère l'ensemble des aidants hors ménage ou uniquement ceux qui aident plus d'une heure par jour.

En Europe du Sud et en Europe de I'Est, les seniors habitant en Espagne, Grèce et Pologne apparaissent significativement moins souvent aidants qu'en France. Ils ne se distinguent cependant plus dès lors que I'on considère les individus aidant plus d'une heure par semaine. Il est intéressant de remarquer que toutes choses égales par ailleurs (en particulier quand on contrôle par la position des seniors vis-à-vis du marché de l'emploi), les Espagnols ne sont pas plus fréquemment aidants « intensifs » qu'en France ${ }^{19}$. L'Italie est en revanche caractérisée par une proportion plus importante de seniors apportant plus d'une heure d'aide par jour.

Au sein des pays d'Europe du Nord, la plus forte implication dans I'aide vue lors de l'analyse statistique précédente n'est plus visible dès lors que I'on adopte un raisonnement « toutes choses égales par ailleurs ».

Les filles apparaissent plus fréquemment aidantes que les fils, surtout lorsque I'on considère les enfants apportant plus d'une heure d'aide par jour.

Par ailleurs, les jeunes seniors, ceux ayant fait des études supérieures ou ceux ne déclarant pas un état de santé « médiocre » aident plus fréquemment leur parent âgé, sans doute de manière "non intensive ». Ces variables ne jouent en effet plus quand il s'agit de la fourniture d'une aide supérieure à une heure par jour. De manière opposée, le fait d'avoir un conjoint n'a un effet négatif visible que pour ceux apportant plus d'une heure d'aide par jour.

Le comportement d'aide semble par ailleurs être influencé par la présence $d^{\prime}$ autres aidants potentiels. En particulier, le fait d'avoir des frères et sœurs tend à diminuer la probabilité d'aider son parent. Au-delà du nombre de frères et sœurs, le rang dans la fratrie semble aussi jouer sur l'implication dans l'aide. Mais, suivant que l'on considère l'ensemble des aidants ou uniquement ceux aidant plus d'une heure par jour, l'effet du rang n'est pas le même. Les aînés sembleraient plus que les autres s'impliquer de manière " non intensive », tandis que les benjamins sembleraient plus que les autres s'impliquer de manière « intensive ».

Le comportement d'aide des seniors est aussi fonction des caractéristiques du parent. Les mères seraient plus souvent aidées que les pères. Cette

19 Nous rappelons que nous avons été contraints d'exclure de l'estimation les seniors cohabitant avec leur parent âgé alors qu'ils représentent près d'un aidant sur deux en Espagne (tableau 1, page 38). 
différence n'est cependant visible que pour l'aide " non intensive », la différence disparaissant dès lors que I'on considère uniquement I'implication "intensive ». L'âge et l'état de santé ont eux aussi un effet significatif sur le comportement d'aide. Les enfants s'impliquent plus fréquemment dans l'aide quand le parent est âgé et caractérisé, selon l'enfant, par un état de santé « médiocre » ou « acceptable ${ }^{20}$.

\section{- L'arbitrage entre aide et emploi}

Qu'en est-il maintenant des interactions entre le fait d'aider un parent âgé et le fait d'exercer une activité professionnelle?

Au regard des résultats de l'estimation 1, travailler et aider apparaissent toutes choses égales par ailleurs comme deux activités indépendantes et ce, quel que soit le groupe de pays considéré. Cela s'explique sans doute par le fait que la majorité des aidants apportent moins d'une heure d'aide par jour, ce qui limite a priori l'existence d'un quelconque effet de l'une des activités sur l'autre.

Le fait de restreindre l'analyse aux interactions entre emploi et aide « intensive » change quelque peu ce constat et introduit des différences entre pays. Les deux activités demeurent indépendantes en Europe du Nord et en Europe continentale. Dans ces deux régions, les seniors ne renonceraient donc pas à leur emploi pour venir en aide à leur parent âgé. Cela les distingue des pays d'Europe du Sud et d'Europe de l'Est, où aide et emploi ne constitueraient pas deux activités indépendantes: dans ces deux régions, le fait d'apporter en moyenne plus d'une heure d'aide par jour à un parent âgé réduirait la probabilité d'exercer une activité professionnelle. L'aide à un parent âgé expliquerait donc en partie le fait que les pays d'Europe du Sud et d'Europe de l'Est soient en moyenne caractérisés par un plus faible taux d'emploi des seniors que leurs voisins continentaux et nordiques. Les pays d'Europe de l'Est présentent cependant une spécificité liée au fait que, toutes choses égales par ailleurs, travailler augmenterait la probabilité d'offrir plus d'une heure d'aide par jour à un parent âgé.

Ce dernier résultat, qui semble propre aux hommes (tableau 5, page 50), révèle sans doute une des limites de l'estimation proposée ici. Nous ne tenons en effet pas compte de l'aide financière que les seniors peuvent apporter à leur parent, alors que celle-ci peut être liée positivement au fait d'exercer une

20 La distance géographique séparant l'enfant de son parent âgé est aussi un déterminant de l'aide. Nous ne l'avons cependant pas intégré comme explicative dans le modèle car elle souffre potentiellement d'un important biais d'endogénéité (Stern, 1995). Il est en effet possible que l'aide soit un déterminant de la distance géographique et non l'inverse, les enfants désireux de s'impliquer dans la prise en charge pouvant se rapprocher de leur parent. 
activité professionnelle. L'aide financière ascendante, assez rare dans la majorité des pays européens ${ }^{21}$, peut apparaître non négligeable en Europe de I'Est, ce qui expliquerait, en supposant que les « aidants en temps » sont aussi fréquemment " aidants en argent», que les individus exerçant une activité professionnelle soient plus fréquemment aidants que les autres en Europe de I'Est. Enfin, une autre limite de l'analyse réside dans le fait que nous ne tenons pas compte ici du nombre d'heures travaillées par les actifs occupés, les individus pouvant renoncer partiellement et non totalement à leur activité professionnelle.

\section{Tableau 6. Estimation simultanée des déterminants de l'aide et de l'emploi}

\begin{tabular}{|c|c|c|c|c|c|}
\hline & \multicolumn{2}{|c|}{ Estimation 1} & \multicolumn{2}{|c|}{ Estimation 2} \\
\hline & & $\begin{array}{l}\text { Aidant hors } \\
\text { ménage } \\
\text { (1) }\end{array}$ & $\begin{array}{l}\text { Actif occupé } \\
\text { (2) }\end{array}$ & $\begin{array}{l}\text { Aidant hors } \\
\text { ménage plus } \\
\text { d'1h/jour (3) }\end{array}$ & $\begin{array}{l}\text { Actif occupé } \\
\text { (4) }\end{array}$ \\
\hline Constante & & $-0,54^{\star \star \star}(0,18)$ & $0,26^{\star \star \star}(0,12)$ & $-1,27^{\star \star \star *}(0,23)$ & $0,28^{\star * *}(0,10)$ \\
\hline \multirow{13}{*}{ Pays } & Autriche & $-0,27^{\star}(0,14)$ & $-0,58^{\star \star \star}(0,15)$ & $0,03(0,18)$ & $-0,57^{\star \star *}(0,15)$ \\
\hline & Allemagne & $-0,04(0,10)$ & $-0,14(0,22)$ & $0,13(0,14)$ & $-0,14(0,11)$ \\
\hline & Suède & $0,10(0,17)$ & $0,70^{\star \star \star}(0,14)$ & $-0,37(0,25)$ & $0,61^{\star *}(0,11)$ \\
\hline & Pays-Bas & $0,08(0,17)$ & $-0,27(0,12)$ & $-0,20(0,28)$ & $-0,35^{\star \star}(0,15)$ \\
\hline & Espagne & $-0,39^{* *}(0,16)$ & $-0,01(0,13)$ & $0,16(0,19)$ & $0,04(0,13)$ \\
\hline & Italie & $0,02(0,13)$ & $-0,29^{\star *}(0,12)$ & $0,40 * *(0,16)$ & $-0,21^{\star}(0,13)$ \\
\hline & France & réf. & réf. & réf. & réf. \\
\hline & Danemark & $0,13(0,16)$ & $0,23(0,15)$ & $-0,42^{\star}(0,24)$ & $0,14(0,11)$ \\
\hline & Grèce & $-0,38^{\star \star \star}(0,14)$ & $-0,06(0,11)$ & $0,13(0,17)$ & $0,01(0,10)$ \\
\hline & Suisse & $-0,12(0,12)$ & $0,37^{\star * *}(0,12)$ & $-0,19(0,17)$ & $0,37^{\star * *}(0,12)$ \\
\hline & Belgique & $0,24^{\star \star \star}(0,09)$ & $-0,21^{\star \star}(0,09)$ & $0,30 * *(0,13)$ & $-0,20^{\star *}(0,10)$ \\
\hline & Rép. tchèque & $-0,1(0,14)$ & $0,07(0,13)$ & $0,13(0,18)$ & $0,11(0,12)$ \\
\hline & Pologne & $-0,39^{\star * *}(0,14)$ & $-0,45^{\star \star *}(0,13)$ & $0,03(0,18)$ & $-0,42^{\star * *}(0,12)$ \\
\hline \multicolumn{6}{|c|}{ Caractéristiques de l'enfant } \\
\hline \multirow{2}{*}{ Sexe } & femme & réf. & réf. & réf. & réf. \\
\hline & homme & $-0,30^{\star \star \star}(0,06)$ & $0,65^{\star \star \star}(0,05)$ & $-0,50^{\star \star \star}(0,08)$ & $0,63^{* * *}(0,05)$ \\
\hline \multirow{3}{*}{ Âge } & {$[50 ; 54]$} & réf. & réf. & réf. & réf. \\
\hline & {$[55,59]$} & $-0,12^{\star}(0,07)$ & $-0,76^{\star \star *}(0,05)$ & $-0,01(0,09)$ & $-0,76^{\star * *}(0,05)$ \\
\hline & {$[60 ; 65]$} & $-0,18(0,14)$ & $-1,93^{\star * *}(0,07)$ & $-0,21(0,16)$ & $-1,92^{\star * *}(0,08)$ \\
\hline \multirow{2}{*}{$\begin{array}{l}\text { Études } \\
\text { supérieu- } \\
\text { res }\end{array}$} & non & réf. & réf. & réf. & réf. \\
\hline & oui & $0,13^{\star *}(0,06)$ & $0,47^{\star * \star}(0,06)$ & $0,08(0,08)$ & $0,47^{\star * *}(0,06)$ \\
\hline \multirow{5}{*}{$\begin{array}{l}\text { État de } \\
\text { santé }\end{array}$} & "médiocre » & $-0,39^{\star \star \star}(0,13)$ & $-1,30^{\star * *}(0,12)$ & $-0,19(0,16)$ & $-1,30^{\star \star *}(0,12)$ \\
\hline & « acceptable » & $-0,07(0,07)$ & $-0,47^{\star \star *}(0,06)$ & $-0,06(0,09)$ & $-0,48^{* * *}(0,06)$ \\
\hline & «bon » & réf. & réf. & réf. & réf. \\
\hline & « très bon » & $0,07(0,06)$ & $0,13^{\star *}(0,05)$ & $-0,05(0,08)$ & $0,13^{\star *}(0,06)$ \\
\hline & " excellent » & $-0,01(0,07)$ & $0,11(0,07)$ & $0,03(0,10)$ & $0,10(0,07)$ \\
\hline \multirow{2}{*}{$\begin{array}{l}\text { A un } \\
\text { conjoint }\end{array}$} & non & réf. & réf. & réf. & réf. \\
\hline & oui & $-0,06(0,05)$ & $-0,06(0,06)$ & $-0,17^{\star *}(0,07)$ & $-0,08(0,06)$ \\
\hline
\end{tabular}

21 Cf. l'étude de Wolff et Attias-Donfut (2007) sur les données de la première vague de l'enquête Share. 
Tableau 6. (suite)

Estimation 1

\begin{tabular}{|l|c|c|c|}
$\begin{array}{l}\text { Aidant hors } \\
\text { ménage (1) }\end{array}$ & $\begin{array}{c}\text { Actif occupé } \\
(2)\end{array}$ & $\begin{array}{l}\text { Aidant hors } \\
\text { ménage plus } \\
\text { d'1h/jour (3) }\end{array}$ & $\begin{array}{c}\text { Actif occupé } \\
\text { (4) }\end{array}$ \\
\hline
\end{tabular}

\begin{tabular}{|c|c|c|c|}
\hline Caractérist & ques de la & & \\
\hline & 0 & réf. & réf. \\
\hline Nombre & 1 & $-0,09^{\star}(0,06)$ & $-0,21^{\star \star *}(0,07)$ \\
\hline de frères & 2 & $-0,19^{* * *}(0,06)$ & $-0,27^{\star \star \star}(0,09)$ \\
\hline & 3 ou plus & $-0,30^{\star \star \star *}(0,07)$ & $-0,29 * * *(0,11)$ \\
\hline & 0 & réf. & réf. \\
\hline Nombre & 1 & $-0,14^{\star \star \star}(0,05)$ & $-0,18^{\star \star *}(0,07)$ \\
\hline de sceurs & 2 & $-0,17^{\star * *}(0,06)$ & $-0,11(0,09)$ \\
\hline & 3 ou plus & $-0,38^{* * *}(0,08)$ & $-0,53^{\star * *}(0,12)$ \\
\hline Aîné de & non & réf. & réf. \\
\hline la fratrie & oui & $0,08^{\star}(0,05)$ & $0,03(0,07)$ \\
\hline Benjamin & non & réf. & réf. \\
\hline fratrie & oui & $0,03(0,06)$ & $0,19^{\star *}(0,08)$ \\
\hline
\end{tabular}

\section{Caractéristiques du parent}

\begin{tabular}{|c|c|c|c|}
\hline \multirow{2}{*}{ Sexe } & femme & réf & réf. \\
\hline & homme & $-0,22^{\star * \star}(0,06)$ & $-0,02(0,09)$ \\
\hline \multirow{5}{*}{ Âge } & ]$-; 79]$ & $-0,21^{\star \star *}(0,07)$ & $-0,23^{\star *}(0,10)$ \\
\hline & {$[80 ; 84]$} & réf. & réf. \\
\hline & {$[85 ; 89]$} & $0,12^{\star \star \star}(0,05)$ & $0,07(0,07)$ \\
\hline & {$[90 ; 94]$} & $0,19^{* \star *}(0,07)$ & $0,19^{* *}(0,10)$ \\
\hline & {$[95 ;+[$} & $0,42^{\star \star \star}(0,11)$ & $0,32^{\star \star}(0,14)$ \\
\hline \multirow{2}{*}{$\begin{array}{l}\text { État de } \\
\text { santé }\end{array}$} & $\begin{array}{l}\text { « médiocre » } \\
\text { ou } \\
\text { « acceptable » }\end{array}$ & $0,22^{\star \star \star}(0,05)$ & $0,45^{\star * *}(0,06)$ \\
\hline & $\begin{array}{l}\text { " bon ", } \\
\text { " très bon » ou } \\
\text { « excellent » }\end{array}$ & réf & réf \\
\hline
\end{tabular}

\section{Interaction entre travail et aide}

\begin{tabular}{|c|c|c|c|c|c|}
\hline \multirow{2}{*}{$\begin{array}{l}\text { Aidant hors } \\
\text { ménage }\end{array}$} & non & & réf & & \multirow{2}{*}{$\begin{array}{c}\text { réf } \\
-0,20(0,33)\end{array}$} \\
\hline & oui & & $-0,05(0,22)$ & & \\
\hline \multirow{2}{*}{$\begin{array}{l}\text { Aidant hors } \\
\text { ménage* Est }\end{array}$} & non & & réf & & réf \\
\hline & oui & & $-0,27(0,26)$ & & $-0,75^{* *}(0,38)$ \\
\hline \multirow{2}{*}{$\begin{array}{l}\text { Aidant hors } \\
\text { ménage* Nord }\end{array}$} & non & & réf & & réf \\
\hline & oui & & $-0,18(0,24)$ & & $-0,02(0,49)$ \\
\hline \multirow{2}{*}{$\begin{array}{l}\text { Aidant hors } \\
\text { ménage* Sud }\end{array}$} & non & & réf & & réf \\
\hline & oui & & $-0,13(0,23)$ & & $-0,68^{*}(0,41)$ \\
\hline \multirow{2}{*}{ Actif occupé } & non & réf & & réf & \\
\hline & oui & $0,14(0,22)$ & & $0,09(0,29)$ & \\
\hline \multirow{2}{*}{$\begin{array}{l}\text { Actif occupé * } \\
\text { Est }\end{array}$} & non & réf & & réf & \\
\hline & oui & $0,16(0,23)$ & & $0,47^{*}(0,29)$ & \\
\hline \multirow{2}{*}{$\begin{array}{l}\text { Actif occupé * } \\
\text { Nord }\end{array}$} & non & réf & & réf & \\
\hline & oui & $0,13(0,21)$ & & $-0,14(0,34)$ & \\
\hline \multirow{2}{*}{$\begin{array}{l}\text { Actif occupé * } \\
\text { Sud }\end{array}$} & non & réf & & réf & \\
\hline & oui & $-0,19(0,20)$ & & $0,26(0,31)$ & \\
\hline
\end{tabular}

Entre parenthèses figurent les écarts-types des coefficients estimés.

${ }^{*}$ significatif au seuil de $10 \%$. ${ }^{* *}$ significatif au seuil de $5 \%$. ${ }^{* * *}$ significatif au seuil de $1 \%$. 


\section{Conclusion}

Les Européens de 50-65 ans semblent concernés à double titre par le vieillissement de la population. Le nombre croissant de personnes âgées dépendantes a tout d'abord un effet direct sur leurs conditions de vie puisqu'ils sont alors plus nombreux à devoir prendre en charge un parent dont l'état de santé nécessite une prise en charge. Ils sont aussi concernés par un effet indirect puisqu'ils sont au niveau européen encouragés à quitter le plus tard possible le marché du travail afin de limiter la pression s'exerçant sur les finances publiques.

L'analyse que nous avons proposée avait pour objectif d'étudier jusqu'à quel point il est possible d'encourager l'activité d'une population qui joue par ailleurs un rôle-clé dans la prise en charge des personnes âgées dépendantes. L'enquête Share apparaît de ce point de vue très utile car elle permet de comparer des pays qui, au regard de l'aide apportée aux parents âgés d'une part, et du taux d'emploi d'autre part, présentent une hétérogénéité relativement forte.

Nos résultats montrent que c'est dans les pays d'Europe du Sud (Espagne, Grèce et Italie) et dans les pays d'Europe de l'Est (Pologne et République tchèque), là où la prise en charge publique de la dépendance est la plus réduite, que les effets négatifs de l'aide se font ressentir sur la participation au marché du travail. Dans les pays d'Europe du Nord (Danemark, Pays-Bas et Suède) et d'Europe continentale (Autriche, Allemagne, Belgique, France, Suisse), là où les dispositifs de prise en charge publique de la dépendance sont, à des degrés divers, les plus développés, I'aide à un parent âgé n'aurait au contraire aucun effet significatif sur la participation des seniors au marché du travail. II semblerait donc que le développement de dispositifs publics visant à soulager les aidants informels soit favorable à l'accroissement de l'emploi des seniors. L'analyse effectuée doit cependant être complétée par une étude plus fine des autres effets de l'aide sur l'activité professionnelle que celui se traduisant par un renoncement pur et simple (réduction du nombre d'heures travaillées, aménagement des horaires, postes de moindre responsabilité, etc.). Une approche qualitative visant à retracer les trajectoires professionnelles des aidants semblerait de ce point de vue particulièrement utile pour compléter l'analyse quantitative proposée ici. 


\section{Bibliographie}

Alborg D., 1998, «Intergenerational transmission of health », American Economic Review, vol. 88, n² 2, p. 265-270.

BereCKI-Gisolf J., LUCKe J., Hockey R. \& Dobson A., 2008, «Transitions into informal caregiving and out of paid employment of women in their 50s », Social Science \& Medicine, n 67 (1), p. 122-127.

BJORN P.A., VuONG Q.H., 1985, «Simultaneous Equations Models for Dummy Endogenous Variables: A Game Theoretic Formulation with application to Labor Force Participation », Working Paper $n^{\circ} 537$, Caltech, Pasadena, CA.

Bolin K. Lindgren B., LUNDBorg P., 2008, "Your next of kin or your own carer? Caring and working among the 50 + of Europe », Journal of Health Economics, vol. 27, p 718-738.

BONSANG E., 2007, « How middle-aged women allocate time and money transfers to their older parents in Europe? » Empirica, vol. 34, p 171-188.

Casado-Marín D., García-Gómez P., López-Nicolás Á., 2008, « Labour and income effects of caregiving across Europe: an evaluation using matching », HEDG Working Paper 08/23.

Carmichael F., Charles S., 1998, «The labour market costs of community care », Journal of Health Economics, n 17, p. 747-765.

Carmichael F., Charles S., 2003, «The opportunity costs of informal care: does gender matter? » Journal of Health Economics, n 22, p. 781-803.

Case A., Lubotsky D., Paxson C., 2002, "Economic status and health in childhood: the origins of the gradient ", American Economic Review, vol. 92, no 5, p. 1308-1334.

CRESPO, 2007, "Caring for parents and employment status of European mid-life women », CEMFI Working Paper.

Devaux M., Jusot F., Trannoy A., Tubeuf S., 2008, " La santé des seniors selon leur origine sociale et la longévité de leurs parents », Économie et statistique, vol. 411, p. 25-46.

DutheIL, 2002, "Les aidants des adultes handicapés », Études et Résultats, $\mathrm{n}^{\circ} 186$, Drees. 
ETtNER S. L., 1995, «The impact of parent care on female labor supply decisions », Demography, vol. 32 (1), p. 63-80.

ETTNER S. L., 1996, «The opportunity cost of elder care», Journal of Human Resources, vol. 31 (1), p. 189-205.

FondATION MÉdÉRIC AlzheImer, 2001, Vivre avec la maladie d'Alzheimer. L'aide aux aidants en France: identifier, comprendre, agir, Ouvrage collectif, Éditions Fondation Médéric Alzheimer.

Fontaine R., Gramain A., Wittwer J., 2007, « Les configurations d'aide familiales mobilisées autour des personnes âgées dépendantes en Europe », Économie et statistique, vol. 403-404, p. 97-115.

Fontaine R., Gramain A., Wittwer J., 2009, « Providing care for an elderly parent: interactions among siblings? » Health Economics, vol. 18 (9), p. 1011-1029.

HeCKMAN J., 1978, "Dummy endogenous variables in a simultaneous equation system », Econometrica, vol. 46, p. 931-959.

Heitmueller A., 2007, "The chicken or the egg? Endogeneity in labour market participation of informal carers in England », Journal of Health Economics, $\mathrm{n}^{\circ} 26$, p. 536-559.

JOHNSON R.W., Lo SASSO A.T., 2000, "The trade-off between hours of paid employment and time assistance to elderly parents at mid-life ", Report from the Urban Institute, Washington D.C.

KRAUTH B.V., 2006, "Simulation-based estimation of peer effects ", Journal of Econometric, vol. 133, p. 243-271.

Le Bihan-Youinou B., MARTin C., 2006, "Travailler et prendre soin d'un parent âgé dépendant », Travail, genre et société, vol. 16, p. 77-96.

MAdDALA G.S., 1983, «Limited-Dependent and Qualitative Variables in Econometrics », Econometric Society Monographs in Quantitative Economics, Cambridge, University Press.

OECD, 2005, « Long-term Care for Older People ».

PezZIN L.E., Shone B.S., 1999, «Intergenerational household formation, labor supply, and informal caregiving: a bargaining approach ", Journal of Human Resources, vol. 34 (3), p. 475-503. 
Soetevent A.R., Kooreman P., 2007, " A discrete-choice model with social interactions: with an application to high school teen behaviour », Journal of Applied Econometrics, vol. 22, p. 599-624.

SteRn S., 1995, "Estimating family long-term care decisions in the presence of endogenous child characteristics », Journal of Human Resources, vol. 30 (3), p. 551-580.

TAMER E., 2003, «Incomplete Simultaneous Discrete Response Model with Multiple Equilibria ", Review of Economic Studies, vol. 70 (1), p. 147-165.

Wolf D.A., Soldo B., 1994, "Married Women's allocation of time to employment and care of elderly parents ", Journal of Human Resources, vol. 29 (3), p. 1259-1276.

WolfF F.-C., ATtias-Donfut C. (2007), « Les comportements de transferts intergénérationnels en Europe », Économie et Statistique, vol. 403-404, p. 117-141. 